\title{
CLASSIFICATION OF PRODUCTS AND SERVICES TO SUPPORT BUSINESS PROCESS ENGINEERING AND E-COMMERCE
}

\author{
Peter J. A. Reusch 1), Pascal Reusch ${ }^{2}$ ) \\ 1) University of Applied Sciences Dortmund, Peter.Reusch@FH-Dortmund.de \\ 2) University of Cologne, ReuschP@t-online.de
}

\begin{abstract}
Decades ago individual approaches to classify products and services had been introduced within companies to unify products and to reduce stocks and costs. Other approaches had been introduced to support international trade and tariff systems. Today new approaches are introduced to support e-commerce and improve business processes. All these approaches are different in the way how to classify, what to classify, and what results to get - and the language they use. The harmonization of all these approaches is very difficult. But especially companies that want to take part in B2B-business need bridges between the different approaches

In this paper we first present a new XML-based system to remove the language barrier within classification systems and to improve data exchange.

In the second part we present an implementation of classification systems based on topic maps according to the XTM standard to implement single classification systems and establish mappings between corresponding classes of different classification systems.
\end{abstract}

Keywords: - Classification, e-Commerce, Topic Map

\section{CLASSIFICATION OF PRODUCTS AND SERVICES FOR E-COMMERCE}

\subsection{INTRODUCTION}

In Europe the most important product and service classification for e-commerce is ecl@ss ${ }^{1}$, built by a network of supporting industries and managed by the Cologne Institute for Business Research ${ }^{2}-$ the authors of this paper are associated to this institute. In America UNSPSC ${ }^{3}$ is the classification system that is generally prefered. A lot of other classification systems exist for special industries and within companies.

eCl@ss features a four level, hierarchical classification key with a keyword index containing 14,000 terms. The hierarchical levels are: segment, main group, group and commodity class. This system provides a structure for classifying all materials and services, with a degree of detail that is well suited to the requirements of the industry to improve procurement and other processes.

In ecl@ss classes are linked to a set of attributes that can be used to describe the products of the class in a uniform way - to improve the communication

${ }^{1}$ www.eclass.de
${ }^{2}$ http://www.iwkoeln.de/
${ }^{3}$ www.unspsc.com/

between suppliers and buyers. In the middle of the year2003 ec1@ss is available in German, English, Spanish, Italian, Czech and French. The Russian version is available as a prototype - at the State Economical University Minsk and at the Fachhochschule in Dortmund we work on this version.

$\begin{array}{ll}20 & \text { Packing material } \\ 21 & \text { Tools, machine tools } \\ 22 & \text { Construction technology } \\ 23 & \text { Machine element, fixing } \\ 24 & \text { Communication technology, office } \\ 25 & \text { Service } \\ 26 & \text { Energy, basic chemicals, aux. agents } \\ 27 & \text { Automation, electrical-engineering, } \\ 27-11 & \text { Lighting installation, device } \\ 27-11-01 & \text { Lighting installation (interior) } \\ 27-11-02 & \text { Lighting installation (outside) } \\ 27-11-03 & \text { Interior light } \\ 27-11-04 & \text { Exterior lamp } \\ 27-11-05 & \text { Explosion proof light } \\ 27-11-06 & \text { Light medium } \\ 27-11-06-01 & \text { Fluorescent light } \\ 27-11-06-02 & \text { Energy saving light } \\ 27-11-06-03 & \text { Lightbulb (fluorescent) } \\ 28 & \text { Automotive technology } \\ 29 & \text { Home economics, technolology } \\ 30 & \text { Operating, cleaning material, }\end{array}$


equipment

$31 \quad$ Polymers

32 Laboratory material, technology

33 Installation (complete, turnkey)

34 Medicine, medical technology

35 Semifinished products,materials

36 Machine, apparatus

$37 \quad$ Pipeline technology

38 Chemical product (inorganic)

39 Chemical product (organic)

40 Occupational safety, accident 41 prevention

Advertising

Fig. 1 - All segments and some main groups, groups and commodity classes of ecl@ss.

Some classes are linked to synonyms. For commodity class 27-11-06-03 lightbulb, light bulb, general purpose lamp (bulb) are keywords synonyms. All keywords can be used to search for classes.

Classes are linked to attribute sets. The commodity class $27-11-06-03$ is linked to attributes like capacity, color of glass body, product type, and voltage. These attributes are used for the specification of a lightbulb in a tender or in an order. If buyer and supplier use the same attribute sets offers, orders and other documents can be selected and handled more or less automatically.

\subsection{THE NEW ECL@SS-XML-JSP- APPROACH}

The ecl@ss-approach used so far separates the different language modules totally. If a German buyer for example selects a class and its attributes based on the German classification and wants to send an order to a Spanish company, he is not able to switch from the class descriptions and attributes in German to the corresponding descriptions and attributes in Spanish - or any other language.

In his master thesis Gerd Rosarius ${ }^{4}$ presented a new system based on XML and JSP to support the classification of products and services according to ecl@ss with additional assistance to work in different languages. This prototype system is also the first step to improve data exchange.

The following figure shows the XMLspecification of the class for lightbulbs. There are "universal" elements that hold the identification and the classification number, and there are elements for specific languages - the descriptions of the lightbulb in Russian, German and English, and keywords in

\footnotetext{
${ }^{4}$ Gerd Rosarius: Entwicklung eines Anwendungssystems zur

Darstellung einer mehrsprachigen Klassifikation von Waren und

Dienstleistungen im e-Commerce basiered auf XML und JSP, Dortmund 2003.
}

several languages.

$$
\begin{aligned}
& \text { <subclass featurelist="2" id="AAB808001" } \\
& \text { revision=" } 001 \text { " version=" } 001 \text { " }> \\
& <\text { identification }>\text { AAB8008 }<\text { /identification }> \\
& <\text { classification no }>\mathbf{2 7 1 1 0 6 0 3}</ \text { classification no }> \\
& <\text { description lang="ru" }>\text { Лампа накаливания } \\
& </ \text { description }> \\
& <\text { description lang="en" }>\text { Lightbulb (fluorescent) } \\
& <\text { description lang="de" }>\text { Glbhlampe } \\
& </ \text { description }> \\
& </ \text { description }>
\end{aligned}
$$

lamp

$$
<\text { keyword lang ="en" }>\text { General purpose }
$$

$$
\begin{aligned}
& <\text { keyword lang ="en" }>\text { (bulbht }<\text { Light } \\
& \text { bulb }</ \text { keyword }>
\end{aligned}
$$

$<$ keyword lang $=$ "de" $>$ Glbhbirne

(Leuchtmittel) $</$ keyword $>$

$<$ keyword lang $=" \mathrm{de} ">$ Allgebrauchslampe

(Glbhbirne) $<$ /keyword $>$

$</$ subclass $>$

Fig. 2 - XML representation of ecl@ss class 27-11-06-03.

Based on this approach the user of ecl@ss-XMLJSP can follow the classification tree in his language. If he comes to the class he needs he can switch at that node to any description that is implemented and can send this description to his foreign partner.

\section{CLASSIFICATION OF PRODUCTS AND SERVICES BASED ON TOPIC MAPS}

XTM provides an XML-based notation for representing information based on topics, and the relationships between topics. A topic map defines a multidimensional topic space - a space in which the locations are topics, and in which the distances between topics are measurable in terms of the number of intervening topics which must be visited in order to get from one topic to another, and the kinds of relationships that define the path from one topic to another. A topic map provides links to any kind of documents including URLs related to the topics mentioned in the topic map. Topic map engines for web browsers are available to navigate through topic maps based on XTM - for example OMNIGATOR $^{\circledR 5}$, USU $^{\circledR 6}$ or EMPOLIS $\mathrm{K} 42^{\circledR 7}$.

\footnotetext{
5 http://www.ontopia.net

${ }^{6}$ www.usu.de
} 


\subsection{A MONO-HIERARCHICAL APPROACH FOR ECL@SS}

Topic maps can be used to implement classification systems. In the first step metadata are defined to prepare the tree for any hierarchical classification. First we start with a monohierarchical representation for ecl@ss. Using the OMNIGATOR ${ }^{\circledR}$ topic map engine and the Internet Explorer $^{\mathbb{B}}$ we can navigate through the frames or the graphical representation of the class hierarchy.

The class hierarchy is based on a general metadata definition for a topic superclass and a topic subclass according to XTM.

$<$ topic id="superclass">

$<$ subjectIdentity $>$

$<$ subjectIndicatorRef

xlink:href="http://www.topicmaps.org/xtm/

1.0/core.xtm\#superclass"/>

$</$ subjectIdentity $>$

$<$ baseName $>$

$<$ baseNameString $>$ superclass $</$ baseNameString $>$

$<$ /baseName $>$

$<$ topic $>$

$<$ topic id="subclass" $>$

$<$ subjectIdentity>

$<$ subjectIndicatorRef

xlink:href="http://www.topicmaps.org/xtm/

1.0/core.xtm\#subclass"/>

$</$ subjectIdentity $>$

$<$ baseName $>$

$<$ baseNameString $>$ subclass $<$ /baseNameString $>$

$<$ baseName $>$

$</$ topic $>$

Fig. 3 - First group of metadata in the topic map.

Thereafter topic types and topics for individual classes can be introduced. eClass classes is the root element for classification tree representing ecl@ss.

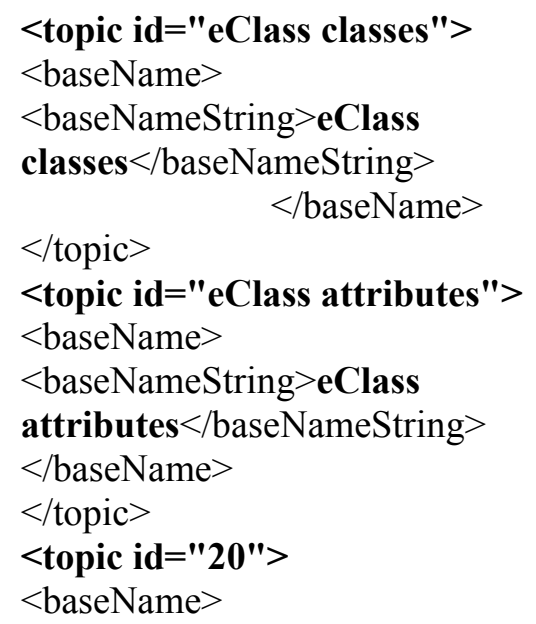

${ }^{7}$ http://k42.empolis.co.uk/

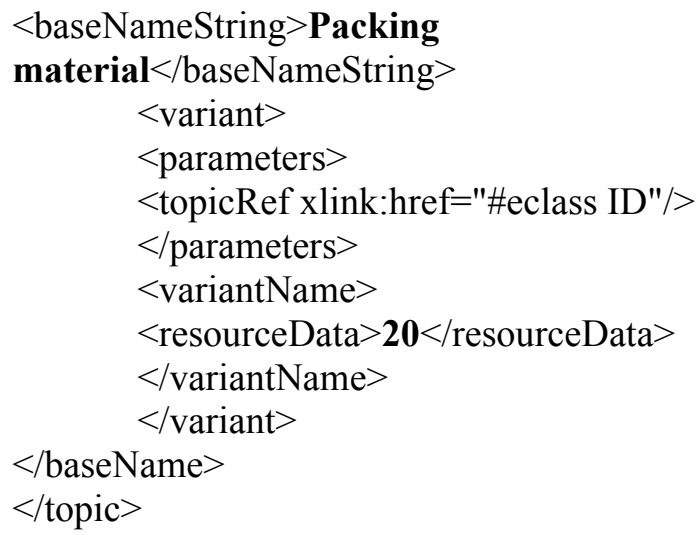

Fig. 4 - Some topics to implement ecl@ss.

The following figures show the OMNIGATORInternet Explorer-presentation of a topic map with a partial representation of ecl@ss.

\section{Topic Map}

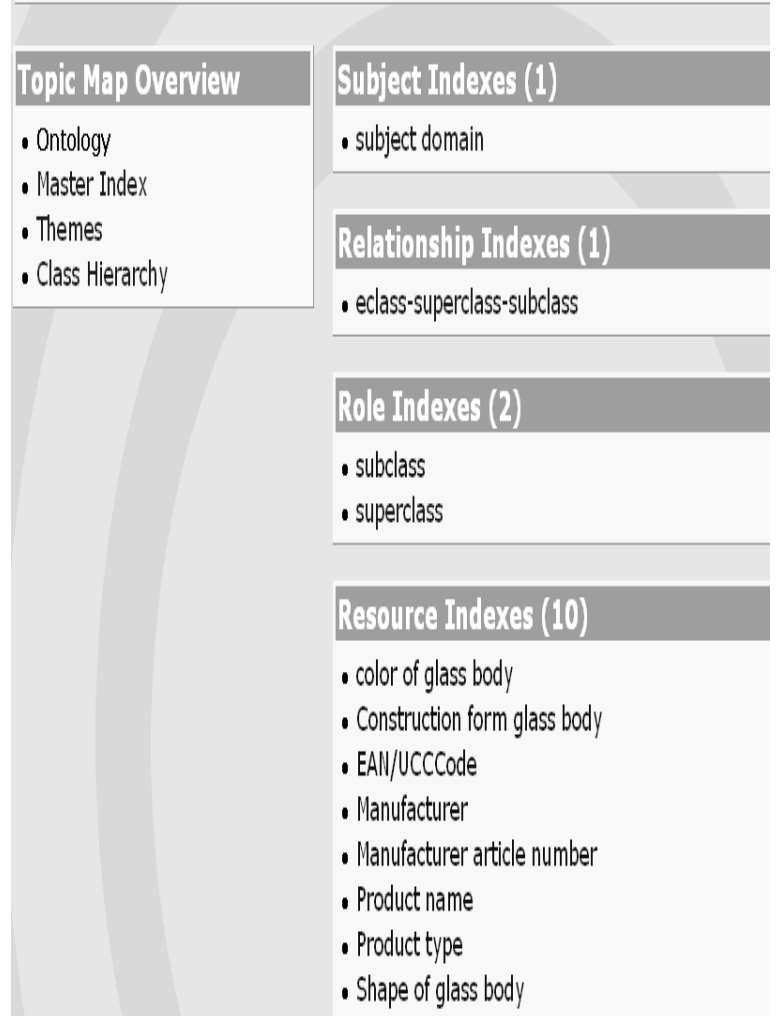

Fig. 5 - Top level representation of a topic map for ecl@ss.

The graphical representation shown above is only available for one and only one hierarchy within the topic map. That is a restriction, but no problem. The graphical representation for classification systems is often useless because of the branching type of these trees, where one node may have about 50 successors. The following approach ignores graphical representations but supports multiple hierarchies. All approaches are open to the representation of synonyms, attributes, ... . 


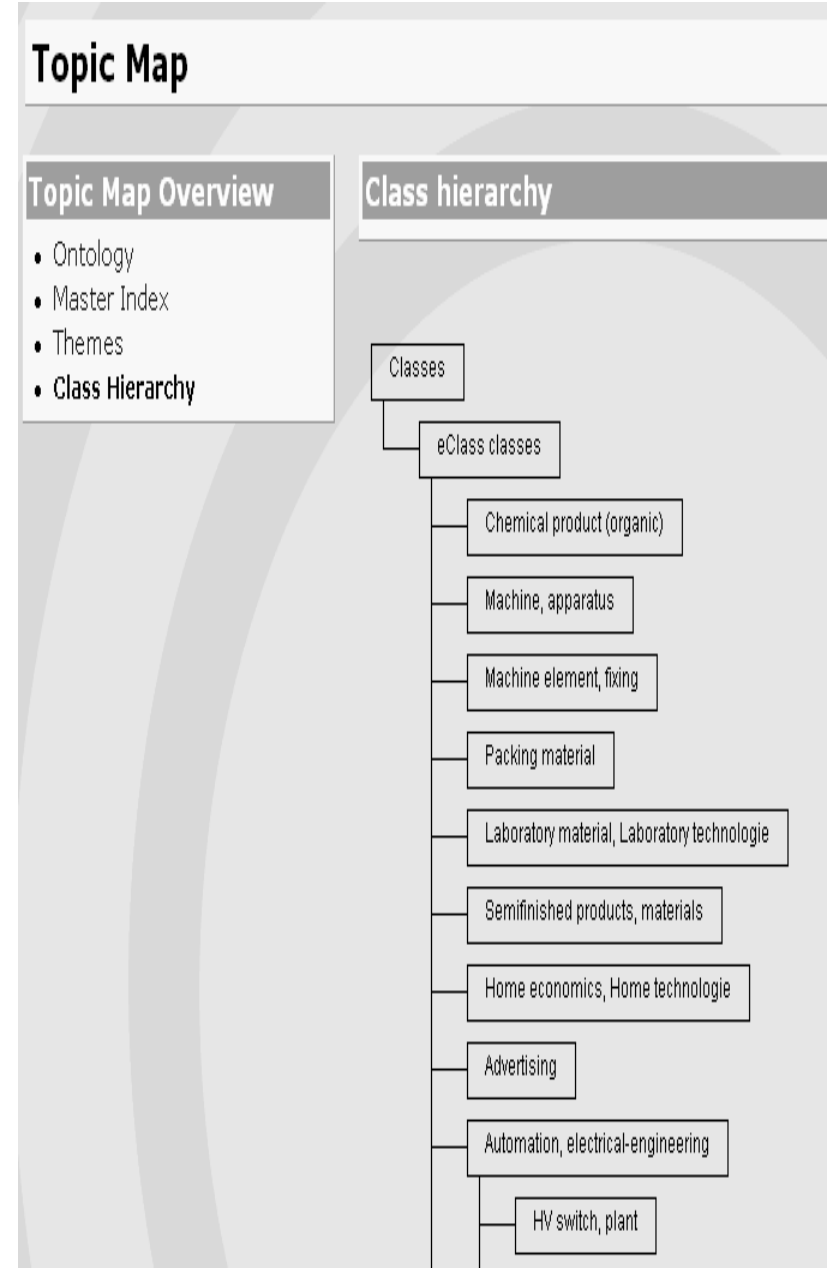

Fig. 6 - Top level representation of a topic map for ecl@ss - graphical class hierarchy.

\section{Lightbulb}

\begin{tabular}{|c|c|}
\hline Names (1) & Internal information (10) \\
\hline $\begin{array}{l}\text { - Lightbulb } \\
\text { o 27-11-06-03 - Scope: I0 } \\
\text { o General purpose light (bulb) - } \\
\text { Scope: Synonym } \\
\text { o Light bulb - Scope: Synonym }\end{array}$ & $\begin{array}{l}\text { - color of glass body } \\
\text { o Clear } \\
\text { o Frosted } \\
\text { o Pink } \\
\text { - Construction form glass body }\end{array}$ \\
\hline & $0 \ldots$ \\
\hline Related subjects (1) & - EAN/UCCCode \\
\hline $\begin{array}{l}\text { - eClass subclass of } \\
\text { o Light medium }\end{array}$ & - Manufacturer \\
\hline & $\begin{array}{l}\text { - Manufacturer article number } \\
\text { - } \ldots \\
\text { - Product name }\end{array}$ \\
\hline
\end{tabular}

Fig.7 - Bottom level representation of the commodity class Lightbulb - subclass of Light medium.

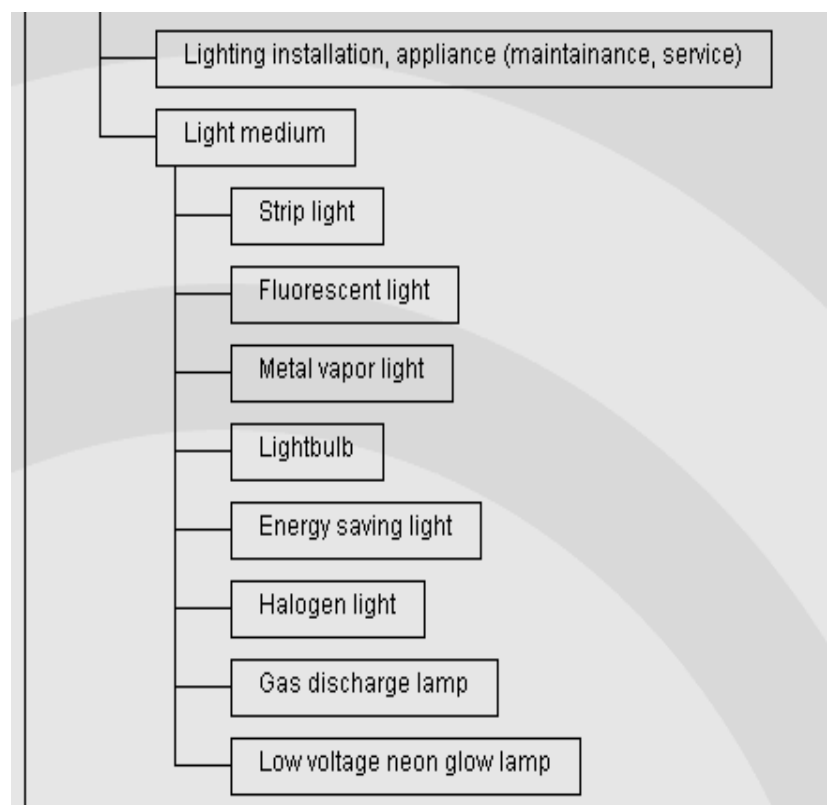

Fig.8 - Graphical representation of the class Lightmedium with some commodity classes.

\subsection{AN OPEN APPROACH FOR UNSPSC}

The UNSPSC-approach for the classification of products and services is quite different from ecl@ss. The following figure shows some segments and some families, classes and commodity classes of UNSPSC.

10000000 Live Plant and Animal Material and Accessories and Supplies

11000000 Mineral and Textile and Inedible Plant and Animal Materials

12000000 Chemicals including Bio Chemicals ..

$13000000 \quad$ Resin and Rosin and Rubber and Foam and Film and Elastomeric Mat.

14000000 Paper Materials and Products

15000000 Fuels and Fuel Additives ...

20000000 Mining Machinery and Accessories

$21000000 \quad$ Farming and Fishing and Forestry and Wildlife Machinery ...

22000000 Building and Construction Machinery

$23000000 \quad$ Industrial Manufacturing and Processing Machinery

24000000 Material Handling and Conditioning and Storage Machinery ..

25000000 Commercial and Military and Private Vehicles and their Accessories ... 


\begin{tabular}{|c|c|}
\hline 26000000 & $\begin{array}{l}\text { Power Generation and Distribution } \\
\text { Machinery and Accessories }\end{array}$ \\
\hline 27000000 & Tools and General Machinery \\
\hline 30000000 & $\begin{array}{l}\text { Structures and Building and } \\
\text { Construction and Manufacturing } \\
\text { Components and Supplies }\end{array}$ \\
\hline 31000000 & Manufacturing Components ... \\
\hline 32000000 & Electronic Components and Supplies \\
\hline 39000000 & $\begin{array}{l}\text { Lighting and Electrical Accessories } \\
\text { and Supplies }\end{array}$ \\
\hline 39100000 & $\begin{array}{l}\text { Lamps and lightbulbs and lamp } \\
\text { components }\end{array}$ \\
\hline 39101600 & Lamps \\
\hline 39101601 & Halogen lamps \\
\hline 39101602 & Medical lamps \\
\hline 39101603 & Solar lamps \\
\hline 39101604 & Alcohol lamps \\
\hline 39101605 & Fluorescent lamps \\
\hline
\end{tabular}

Fig. 9 - Some segments and some families, classes and commodity classes of UNSPSC

Most segments in UNSPSC have no corresponding element in ecl@ss - for example 10000000. Some parts of the classification trees are similar, for example the parts for lamps and light media. But it is really a hard problem for those who need both classification systems to build the bridges between them.

A first topic map-approach for UNSPSC is available http://www.techquila.com/tmsamples/xtm/unspsc/ unspsc_11.zip. There are topic types for segments, families, classes and commodities, and topics for the individual occurrences of theses types.

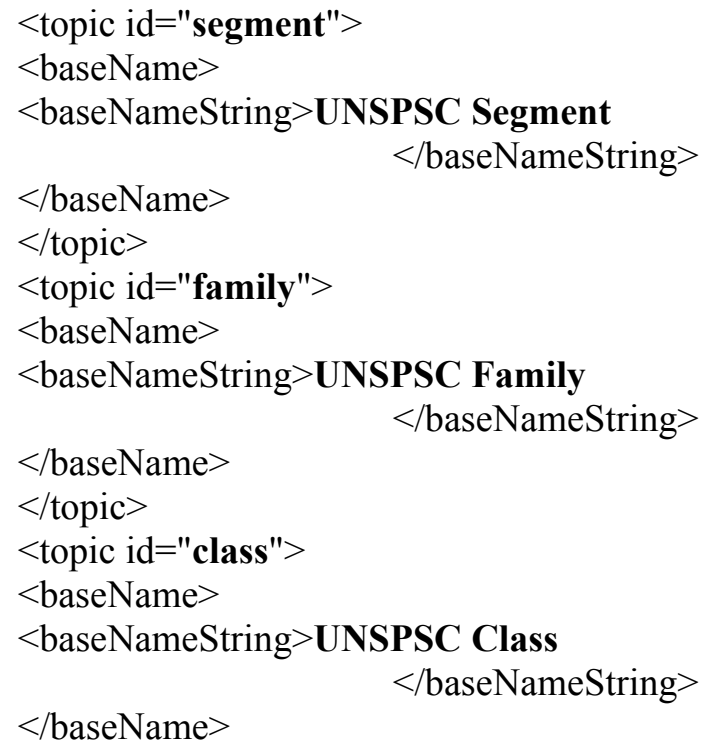

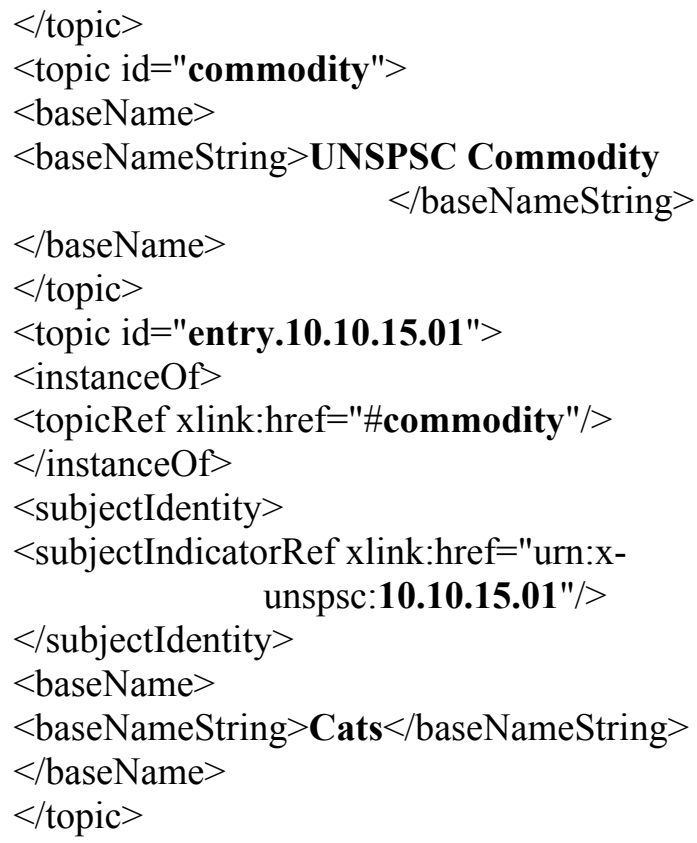

Fig. 10 - Topics for UNSPSC.

There are topics to define association types, for example the following topic to define an association type between segments and families.

$<$ topic id="assoc-segment-family" >

$<$ baseName $>$

$<$ baseNameString $>$ UNSPSC Segment Consists Of Families $</$ baseNameString $>$

$</$ baseName $>$

$<$ baseName $><$ scope $>$

$<$ topicRef xlink:href="\#segment" $></$ scope $>$

$<$ baseNameString $>$ Families $<$ /baseNameString $>$

$<$ /baseName $>$

$<$ baseName $><$ scope $>$

$<$ topicRef xlink:href="\#family" $></$ scope $>$

$<$ baseNameString $>$ Belongs To Segment

$</$ baseNameString $></$ baseName $>$

$</$ topic $>$

Fig. 11 - Topic for an association type.

There are associations defined according to the topic types, for example the following association between segment 10000000 Live Plant and Animal Material and Accessories and Supplies and family 10100000 Live Animals.

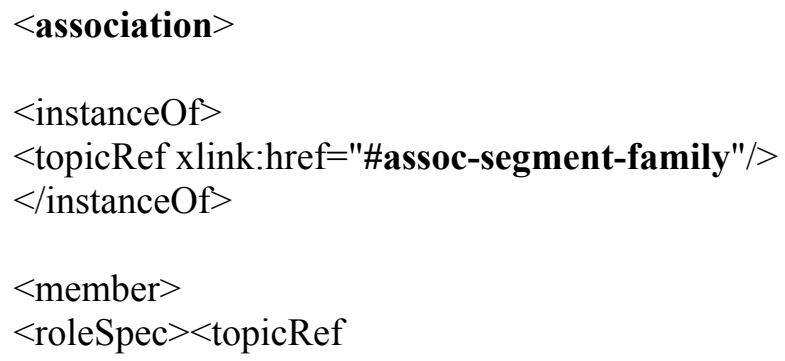


xlink:href="\#segment"/></roleSpec $>$

$<$ topicRef

xlink:href="\#entry.10.00.00.00" $/></$ member $>$

$<$ member $>$

$<$ roleSpec $><$ topicRef

xlink:href="\#family" $></$ roleSpec $>$

$<$ topicRef

xlink:href="\#entry.10.10.00.00" $/><$ member $>$

$</$ association $>$

Fig. 12 - Topic for an association.

The following figure shows the OMNIGATOR ${ }^{\circledR}$ view of the complete topic map for UNSPSC - top level view.

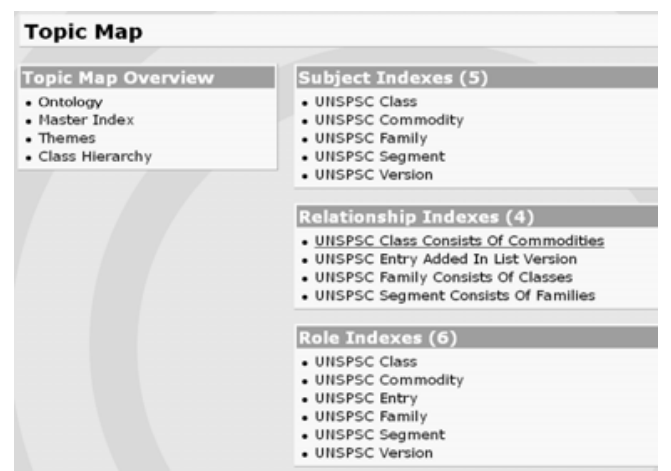

Fig. 13 - UNSPSC topic map OMNIGATOR ${ }^{\circledR}$ view.

The following figure shows the OMNIGATOR ${ }^{\circledR}$ view of the UNSPSC segment 39000000.

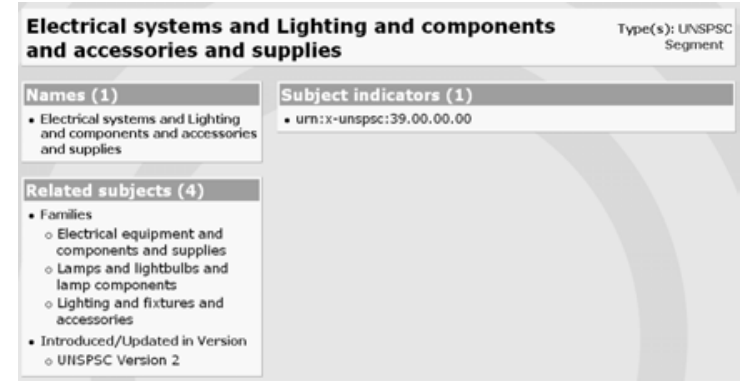

Fig. 14 - UNSPSC topic map OMNIGATOR ${ }^{\circledR}$ view detail.

This approach is appropriate for UNSPSC, but not appropriate if we want to build a topic map including several classification systems and bridges between corresponding elements. To open the topic map we have to find a unique way to define the topics of all included classification systems. The following figure shows a first step to get unique topic types.

$<$ topic id="unspsc-segment" $><$ baseName $>$

$<$ baseNameString $>$ UNSPSC Segment

$<$ /baseNameString $></$ baseName $></$ topic $>$
$<$ topic id=" unspsc-family" $><$ baseName $>$

$<$ baseNameString $>$ UNSPSC Family

$</$ baseNameString $><$ /baseName $></$ topic $>$

$<$ topic id=" unspsc-class" $><$ baseName $>$

$<$ baseNameString $>$ UNSPSC

Class $<$ /baseNameString $>$

$<$ /baseName $><$ /topic $>$

$<$ topic id=" unspsc-commodity" $><$ baseName $>$

$<$ baseNameString $>$ UNSPSC Commodity

$</$ baseNameString $><$ /baseName $><$ /topic $>$

$<$ topic id="eclass-segment" $><$ baseName $>$

$<$ baseNameString $>$ eclass Segment

$</$ baseNameString $><$ /baseName $><$ /topic $>$

$<$ topic id="eclass - main-group" $><$ baseName $>$

$<$ baseNameString $>$ eclass Main Group

$</$ baseNameString $><$ /baseName $></$ topic $>$

$<$ topic id=" eclass-group" $><$ baseName $>$

$<$ baseNameString $>$ eclass

Group $</$ baseNameString $>$

$<$ baseName $><$ /topic $>$

$<$ topic id=" eclass-commodity" $><$ baseName $>$

$<$ baseNameString $>$ eclass Commodity

$<$ /baseNameString $><$ /baseName $><$ /topic $>$

Fig. 15 - Unique topic types.

Based on these topic types different association types can be defined to build different kind of bridges between corresponding elements of various classification systems.

$<$ topic id="assoc-unspsc-commodity-eclasscommodity">

$<$ baseName $>$

$<$ baseNameString $>$ Corresponding Commodities

UNSPSC-eclass $</$ baseNameString $>$

$<$ baseName $>$

$<$ baseName $><$ scope $>$

$<$ topicRef

xlink:href="\#eclass-

commodity" $/></$ scope $>$

$<$ baseNameString $>$ eclass Commodity

$</$ baseNameString $><$ /baseName $>$

$<$ baseName $><$ scope $>$

$<$ topicRef

xlink:href="\#unspsc-

commodity"/ $></$ scope $>$

$<$ baseNameString $>$ UNSPSC Commodity

$<$ /baseNameString $><$ /baseName $>$

$</$ topic $>$

Fig. 16 - Association type

Based on such association types concrete links 
between corresponding classes can be defined.

\section{CONCLUSION}

\subsection{RESULTS}

XML gives us the key to use classification systems in a multilingual environment. Topic maps give us the key to a representation of classification systems supporting associations between different classification systems.

This result is important to support "standards" like UNSPSC or ecl@ss, it is even more important today to support mappings between in-housesystems and "standards". In-house-systems are used in any bigger company to support workflows and reduce stocks and costs. It is a hard job and it will take a lot of years to convert from the in-housesystem to a "standard". And most companies will not even start to convert now, because all "standards" are still weak in some aspects and the evolution of the "standards" is going on and in some aspects is far away from the level some companies need. So in the time of globalization and mergers the mapping of in-house-systems and "standards" is necessary to support business processes and ecommerce.

The topic map approach is appropriate for all classification systems used. For a single classification system like ecl@ss about $10^{5}$ topics are necessary to implement the system - language versions implemented as variants and separated by scopes.

That is within the range of topic map engines and similar approaches. So that is a key to solve the problem to integrate different classification systems and different language versions.

An essential point is that topic maps are appropriate for a precise description of complex topics and associations with a lot of different roles and many kinds of relations.

\subsection{FURTHER RESEARCH}

There are so many classification systems for products, processes, services, patents, job etc. one has to use almost every day in a bigger company for customs documents or patent registration ${ }^{8}$ or the statistical organizations ${ }^{9}-$ and they all are different - and there are no appropriate mappings between them. Even the mappings between UNSPSC and the Harmonized Classes of the World Customs Organization $^{10}$ failed so far $^{11}$. Both classification

\footnotetext{
${ }^{8} \mathrm{http}: / /$ www.european-patent-office.org/

${ }^{9} \mathrm{http}: / /$ europa.eu.int/comm/eurostat/

${ }^{10} \mathrm{http}: / / \mathrm{www}$. wcoomd.org/ie/En/en.html

${ }^{11} \mathrm{http} / / / \mathrm{www} . \mathrm{eccma} . \mathrm{org} / \mathrm{unspsc} / \mathrm{crosswalk} . \mathrm{htm}$
}

systems have common roots - but passed through an evolution that made the systems incompatible.

A unification of these classifications will not come within our generation. The structure of the classification systems is based on different needs and interests. The Harmonized Classes of the WCO for example do no include services because one does not need that for customs documents. And a lot of branches starting at similar segments are expanded in different ways.

If we are not able to unify these classification systems, we must build mappings between them. For mass mappings we need high sophisticated methods and tools - classifiers, text miners, a new kind of dictionaries, etc.

\section{REFERENCES}

[1] Peter J. R. Reusch e.a.. Klassifikation von Waren und Dienstleistungen. Dortmund 2003.

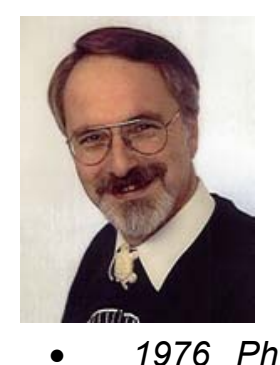

Peter J. A. Reusch

- 1950 born in Bruehl close to Cologne (Germany), on September $26^{\text {th }} 1950$

- 1974 Diploma in

Mathematics

Theses on Complexity Classes of Elementary Functions

(Computer

University of Bonn

Science)

Theses on: Generalized Lattices as Fundamentals for Retrieval Models, Multi-dimensional Developmental System, and the Evaluation of Fuzziness

- 1976 - 1988 Head of the Department for Information Systems at Rheinische Braunkohlenwerke

- 1981 - 1991 Lecturer at the Institute for Computer Science at the University of Bonn

- 1988 - until today Professor for Business Informatics and Management at the University of Applied Sciences - Fachhochschule - Dortmund

- 1989 - until today CEO of IBIES (Institute + Corporation)

- 1990 - 1996 Dean of the Management Faculty of the University of Applied Sciences Fachhochschule - Dortmund

- 1991 - 1997 Professor for Business Informatics at the University of Stettin (Poland) part time

- 1995 Doktor oek. nauk h.c. (ph.d.) at the State Economical University in Minsk (Belarus)

- 1995 - 1998 Visiting Professor at the State University New York - University and College at Buffalo - Summer Sessions July/August 1995, 1996, 1997, 1998

- 1996 Doktor oek. nauk h.c. (ph.d.) University of Latvia, Riga 
- 1999 - until today Professor at the State Economical University in Minsk (Belarus) - part time - 2001 - until today Dean of the Management Faculty of the University of Applied Sciences Fachhochschule - Dortmund

\section{Pascal Reusch was born: 18.05.1980 \\ Education: \\ Diploma in \\ Information Systems from the University of Cologne Diploma Thesis: Support of e-learning processes with Topic Maps}

Since 2003: Working as a research associate at the Production Management Department of University Duisburg-Essen

Writing a dissertation on: "Coordination of hierachical production planning systems" 